University of Nebraska - Lincoln

DigitalCommons@University of Nebraska - Lincoln

Agronomy \& Horticulture -- Faculty Publications

Agronomy and Horticulture Department

2019

History of Grass Breeding for Grazing Lands in the Northern Great Plains of the USA and Canada

\author{
Kenneth P. Vogel \\ University of Nebraska-Lincoln, kvogel1@unl.edu \\ John Hendrickson \\ USDA Northern Great Plains Research Laboratory, john.hendrickson@ars.usda.gov
}

Follow this and additional works at: https://digitalcommons.unl.edu/agronomyfacpub

Part of the Agricultural Science Commons, Agriculture Commons, Agronomy and Crop Sciences Commons, Botany Commons, Horticulture Commons, Other Plant Sciences Commons, and the Plant Biology Commons

Vogel, Kenneth P. and Hendrickson, John, "History of Grass Breeding for Grazing Lands in the Northern Great Plains of the USA and Canada" (2019). Agronomy \& Horticulture -- Faculty Publications. 1282. https://digitalcommons.unl.edu/agronomyfacpub/1282

This Article is brought to you for free and open access by the Agronomy and Horticulture Department at DigitalCommons@University of Nebraska - Lincoln. It has been accepted for inclusion in Agronomy \& Horticulture -Faculty Publications by an authorized administrator of DigitalCommons@University of Nebraska - Lincoln. 


\title{
SiM
}

\section{History of Grass Breeding for Grazing Lands in the Northern Great Plains of the USA and Canada}

\author{
By Kenneth P. Vogel and John Hendrickson
}

\section{On the Ground}

- In the early 1930s there were millions of acres of extensively degraded grazing lands and abandoned and eroded cropland in the Northern Plains of the United States and Canada.

- Grass breeding and plant materials programs were established by both the US and Canadian governments and cooperating universities to develop revegetation materials.

- Efforts of a small number of research locations and people resulted in grass cultivars or varieties that were used to revegetate and preserve the soil on millions of acres of land.

- This is a brief history of the people, agencies, and universities that developed these cultivars that restored and increased the productivity of grasslands in the Northern Plains.

Keywords: grasses, breeding, conservation, rangeland, pastures, cropland.

Rangelands 41(1):1-16

doi 10.1016/j.rala.2018.11.006

(c) 2018 The Society for Range Management.

This document is a U.S. government work and is not subject to copyright in the United States.

Grass Breeding, Cultivars or Varieties, Seed Certification, and Their Importance

A plant variety is a group of plants within a species that are distinct in one or more aspects from other plants of the species. There are naturally occurring botanical varieties. A cultivar is a "cultivated variety" that consists of plants that have been selected or bred by plant breeders for specific characteristics and are maintained through seed increase generations as true-breeding populations of plants. Grass cultivars developed by the first grass breeders were improvements over wild harvest populations of specific grasses for several traits of economic importance. The second and subsequent generations of cultivars represent breeding improvements over the initial cultivars. It needs to be noted that developing and testing perennial grass cultivars and getting the improved plant material into seed production for use in revegetation can take up to 20 years or more as shown in Table 1.

Besides developing grass cultivars, it was also necessary to develop seed certification systems for grasses. The seed certification process ensures the genetic identity and purity of a cultivar. In other words, it ensures the seed in a bag of certified seed is the true seed of the cultivar and not some other seed of the species. This is very important for perennial grasses where the resulting grass stand is often expected to last 20,30, or more years. The expected long life of stands is also the reason why ranchers and farmers should buy certified seed of the best cultivars available at the time of planting. Seed certification is a truth-in-marketing and consumer protection procedure that is needed simply because some people cheat. In both the United States and Canada, seed certification procedures and standards are based on both national and state or province laws and are managed by seed certifying agencies.

\section{The Northern Great Plains and Their Settlement by Euro-Americans}

The prairie and steppe (or plains) ecoregions of the Northern Great Plains includes the states of Nebraska, South and North Dakota, and the areas of Wyoming and Montana east of the Rocky Mountains and the same ecoregions of the Alberta and Saskatchewan provinces of Canada. ${ }^{1}$ For the purpose of this report, the Kansas/Nebraska border $\left(40^{\circ} \mathrm{N}\right.$ latitude) is the southern boundary of the Northern Great Plains. Prior to settlement by people of largely European ancestry, this area was vast, grazed grassland with bison (buffalo) the dominant large herbivore. The Northern Great Plains were primarily settled following the American Civil War from about 1865 to $1900 .^{2,3}$ The settlement proceeded from the higher precipitation areas in the east to the more arid western regions. Crop production was the main focus for the settlers in the eastern part of the region while a ranching 
Table 1. Development phases and timetable for a perennial grass breeding and development program

\begin{tabular}{|c|c|c|c|c|}
\hline Phase & Year 1 & Year 2, 3, or longer & Year 4 or later & Year 5 or later \\
\hline $\begin{array}{l}\text { Phase 1: } \\
\text { Plant materials } \\
\text { (germplasm) } \\
\text { collection or } \\
\text { acquisitions }\end{array}$ & $\begin{array}{l}\text { Establish plant } \\
\text { materials } \\
\text { evaluation } \\
\text { nurseries }\end{array}$ & $\begin{array}{l}\text { Evaluate plant } \\
\text { materials for } \\
\text { persistence, forage } \\
\text { yield, quality, and } \\
\text { other traits }\end{array}$ & $\begin{array}{l}\text { Identify superior } \\
\text { accessions and/or plants } \\
\text { within accessions and } \\
\text { seed harvest or move } \\
\text { selected plants to } \\
\text { crossing blocks }\end{array}$ & $\begin{array}{l}\text { Harvest seed and use } \\
\text { in Phase } 2, \text { Phase } 3 \text {, } \\
\text { or Phase } 5 \text { depending } \\
\text { on status and quality } \\
\text { of plant materials and } \\
\text { objectives }\end{array}$ \\
\hline $\begin{array}{l}\text { Phase } 2 \text { : } \\
\text { Breeding } \\
\text { generations } \\
2,3,4 \text {, or more }\end{array}$ & $\begin{array}{l}\text { Establish selection } \\
\text { nurseries using } \\
\text { seed of selected } \\
\text { and intermated } \\
\text { plants }\end{array}$ & $\begin{array}{l}\text { Evaluate plant } \\
\text { materials for } \\
\text { persistence, forage } \\
\text { yield, quality, etc. }\end{array}$ & $\begin{array}{l}\text { Identify superior plants, } \\
\text { move to crossing blocks, } \\
\text { harvest seed }\end{array}$ & $\begin{array}{l}\text { Harvest seed and use } \\
\text { in next breeding } \\
\text { generation in Phase } \\
2 \text { and in Phase } 3\end{array}$ \\
\hline $\begin{array}{l}\text { Phase 3: } \\
\text { Small plot trials, } \\
\text { single location } \\
\text { or multi-location } \\
\text { regional trials }\end{array}$ & $\begin{array}{l}\text { Plant replicated } \\
\text { small plot trials }\end{array}$ & $\begin{array}{l}\text { Harvest trials; } \\
\text { evaluate stands for } \\
\text { persistence, } \\
\text { diseases, and other } \\
\text { traits; evaluate } \\
\text { forage quality }\end{array}$ & $\begin{array}{l}\text { Summarize data; select } \\
\text { best experimental strains } \\
\text { for release or advance } \\
\text { to Phase } 4 \text {; using seed } \\
\text { from Phase } 1 \text { or } 2 \\
\text { crossing blocks, plant } \\
\text { seed increase nurseries }\end{array}$ & $\begin{array}{l}\text { Harvest seed from } \\
\text { seed increases } \\
\text { (Breeder seed) and } \\
\text { use in Phase } 4 \text { and } \\
\text { Phase 5; multiple } \\
\text { harvest years }\end{array}$ \\
\hline $\begin{array}{l}\text { Phase 4: } \\
\text { Grazing trials } \\
\text { or field scale } \\
\text { trials }\end{array}$ & $\begin{array}{l}\text { Plant pasture } \\
\text { or field trials }\end{array}$ & $\begin{array}{l}\text { Grazing trials or } \\
\text { field trial harvests }\end{array}$ & $\begin{array}{l}\text { Summarize data and } \\
\text { make release decision }\end{array}$ & $\begin{array}{l}\text { Harvest seed from } \\
\text { seed increase } \\
\text { nurseries (Breeder } \\
\text { seed) }\end{array}$ \\
\hline $\begin{array}{l}\text { Phase 5: } \\
\text { Cultivar release } \\
\text { and seed } \\
\text { production }\end{array}$ & $\begin{array}{l}\text { Breeder seed lots } \\
\text { used to establish } \\
\text { Foundation } \\
\text { seed field }\end{array}$ & $\begin{array}{l}\text { Foundation seed } \\
\text { field harvest; fields } \\
\text { harvested many } \\
\text { years; seed provided } \\
\text { to certified } \\
\text { seed growers }\end{array}$ & $\begin{array}{l}\text { Certified seed growers } \\
\text { plant certified seed fields }\end{array}$ & $\begin{array}{l}\text { Certified seed fields } \\
\text { harvested; seed sold } \\
\text { to farmers and } \\
\text { ranches for new } \\
\text { grassland plantings }\end{array}$ \\
\hline
\end{tabular}

Note: A plant breeder will typically have breeding work in progress on several populations of the same species and will often be conducting breeding work on two or more species. The collection and evaluation of new plant collections or acquisitions is an on-going process in most programs.

industry developed in the west, and mixed crop and cattle operations were the norm in the middle zone.

When the Northern Plains were first settled, there was abundant forage for the settlers' horses and cattle. Farmers plowed portions of their farms for crops and left the remainder in grasslands for use by their livestock. The crops that they brought with them such as wheat, oats, and corn were adequately adapted to the region, but the farming systems that they brought with them were not. These systems included annual plowing followed by clean tillage and cultivation for weed control, which left the soil bare and subject to wind and water erosion. Grain crops, such as wheat, were in high demand before and after World War I. As a result, additional millions of acres of native grasslands that were marginal for crop production were plowed during the period of 1905 to 1920 and used for grain production. ${ }^{4-6}$ Prices for grain crops were high and climatic conditions were favorable for grain crop production during this period.

Although it varied with latitude, the perennial prairie grasses were largely warm-season grasses that did not have the growth patterns of the cool-season pasture grasses to which the settlers were accustomed. ${ }^{7}$ Warm-season grasses start growing 4 to 6 weeks later in the spring than cool-season grasses and go dormant when temperatures drop in autumn. As a result, the native pastures and rangelands were often severely overgrazed and in poor condition because the settlers did not know how to manage these grasslands. The settlers were accustomed to continuously grazing cool-season grasses beginning in early spring through late autumn, which adversely affected native warm-season grasses. Because of the bad management practices used on both crop and grazing lands in this region, the conditions were set for an ecological disaster.

\section{Northern Great Plains of the 1930s: Soil, Crop- land, Rangeland, and Pasture Problems}

The severe droughts in the United States and Canada during the period of 1928 to 1937, along with poor crop and grazing land management practices being used in the Great Plains, triggered the ecological and human disaster known as the "Dust Bowl." 8 Although the "Dust Bowl" is often associated with the Southern Plains states, it also had a severe effect on the Northern Plains of the United States and Canada. ${ }^{5,6,9}$ By the mid-1930s, it was obvious that millions of 
acres of land, including overgrazed rangeland in the former prairie and plains states of the United States and Canadian provinces, needed to be restored to grasslands to preserve the soil and convert the agriculture of the region into more sustainable systems as indicated by Secretary of Agriculture Henry Wallace's 1935 Report to the President of the United States, which was published in the 1936 Yearbook of Agriculture. ${ }^{9,10}$ In this report, Wallace points out the need for improved grasses and indicates grass improvement work had been initiated. The need for this research was clearly apparent on 27 April 1935 when a huge dust cloud engulfed Washington, DC during the testimony of Hugh H. Bennett, director of the Soil Erosion Service, to the Senate Public Lands Committee. ${ }^{11}$ Funds were appropriated for the establishment of the Soil Conservation Service (SCS), USDA in 1935 and its associated production and observation plant nurseries. ${ }^{11}$ H.H. Bennett became the director of the SCS. Funding was also made available for USDA grass breeding research. In 1936, the USDA initiated grass breeding research throughout the United States; in the Northern Plains, breeding programs were located at Lincoln, Nebraska and Mandan, North Dakota. ${ }^{12}$

Since 1935, a small number of research locations and scientists in the Northern Great Plains of the United States and Canada have developed over 80 cultivars of 18 different grass species (see Tables 2 and 3) that are adapted to specific areas of the Northern Plains and have been used to revegetate millions of acres of land. These grasses not only led to major achievements in soil conservation but also led to improvements in grazing land productivity. This is a brief history of the people and agencies that made this achievement possible.

\section{Pioneering Perennial Grass Evaluation and Production Research}

Although national initiatives to reseed the eroded lands with grasses had been implemented, there were major problems of seed availability and effective establishment methods that had to be addressed. The only grasses for which seeds had previously been available for seeding pastures in the United States were timothy (Phleum pratense) and orchardgrass (Dactylis glomerata), which had not survived the droughts. ${ }^{7}$ There were problems with using seed from native species and grasslands. Native grasslands had been abundant and common, and as a result almost no information was available on their seed production and establishment. Only limited amounts of seed could be obtained from native harvests in good years and none in drought years. ${ }^{13,14}$

Research was initiated on native grasses beginning in the mid-1930s but a more immediate solution to the revegetation problem was needed. Because of work by a few pioneering grassland scientists, there were two main introduced grasses (crested wheatgrass and smooth bromegrass), which been proven to be broadly adapted to the western and eastern areas, respectively, of the Northern Great Plains. ${ }^{7}$ These two grasses had survived the droughts and also had superior seed and forage production capability in comparison to other available grasses, and were easily planted with available equipment. As a result, these two grasses were the main species that were used in the initial grassland plantings to stabilize the soils of the Northern Great Plains following the1930s drought period. These two introduced grasses are from areas of Europe and Asia that are ecosystem analogs of the Prairie and Temperate Steppe ecoregions of the Northern Great Plains. ${ }^{1}$

Crested wheatgrasses were first introduced into North America in 1892 by N.E. Hansen (Fig. 1) of the South Dakota Experiment Station. ${ }^{4,15}$ Hansen was born in Denmark in 1866 and immigrated with his family to the United States where his family lived in Iowa. ${ }^{16}$ He received BS and MS degrees from Iowa State College and in 1895, he went to South Dakota State College at Brookings, South Dakota, as Professor of Horticulture. Because of his knowledge of plants and languages he was contracted by USDA to do plant exploration and collection. ${ }^{16} \mathrm{In}$ his first collection trip in 1897 to 1898 , he collected five railroad cars of several hundred kinds of grains, grasses, and plants from Russia, Turkestan, China, Siberia, and Transcaucasia. Hansen received a second importation of crested wheatgrass in 1906 from Russia and he made another collection expedition in 1908 for the USDA where additional crested wheatgrass accessions were imported into the United States. ${ }^{15}$ The seed of these grasses were distributed by the Division of Seed and Plant Introduction to experiment stations for testing. ${ }^{17}$

The first known research planting of the crested wheatgrass accessions was at the USDA research station near Newell, South Dakota in 1908. A USDA agronomist at the station, A.C. Dillman, maintained the crested wheatgrass plantings from 1908 to 1915. He harvested and supplied seed to other stations including what is now the USDA Northern Great Plains Laboratory at Mandan, North Dakota and to Canadian researchers at the University of Saskatchewan, Saskatoon, Sask.4,15 Plantings made in 1915 at Mandan, North Dakota by Johnston T. Sarvis were the foundation from which the initial cultivar released in the United States was developed and were the seed sources for seed that was widely distributed to experiment stations and farms in the Dakotas, Montana, and Wyoming from 1920 to $1923.4,15$ The distributed seed was from four of the better selections. ${ }^{4}$ The first pasture trial was established in 1923 at the Ardmore, South Dakota field station and performance trials with dairy cattle were successful. ${ }^{4}$ The first grazing trial with beef cattle was established at Mandan, North Dakota in $1932 .{ }^{15}$ Commercial seed was being produced by farmers in North Dakota and Montana by 1929. ${ }^{4,15}$ In the 25 -year period between when crested wheatgrass was first introduced into the United States and the mid-1930s, agronomists at several experiment stations, primarily the Northern Great Plains Laboratory at Mandan, had thoroughly tested the grass, proved that it could survive even under severe drought conditions, and developed the basic agronomic information needed for seed production, establishment, and use in grazing lands.

In Canada, crested wheatgrass research began in 1916 at Saskatoon, Saskatchewan, by Lawrence E. Kirk, who at that time was a graduate assistant. ${ }^{4}$ Seed was obtained from the USDA from the early collections of Hansen and from other 
Table 2. Cultivars of introduced grasses developed and released for use in the Northern Plains of the United States and Canada, their principal developing agency and location, plant breeder or developer, and adaptation region ${ }^{19,34,35,36}$

\begin{tabular}{|c|c|c|c|c|c|}
\hline \multirow{2}{*}{$\begin{array}{l}\text { Species/ } \\
\text { Cultivar" }\end{array}$} & \multirow{2}{*}{$\begin{array}{l}\text { Agency or } \\
\text { University }\end{array}$} & \multirow{2}{*}{$\begin{array}{l}\text { Breeder or } \\
\text { Developer }\end{array}$} & \multirow{2}{*}{$\begin{array}{l}\text { Released } \\
\text { Year }\end{array}$} & \multicolumn{2}{|c|}{ Adaptation Region } \\
\hline & & & & Ecoregion $^{+}$ & $\begin{array}{l}\text { Hardiness } \\
\text { Zone }^{\ddagger}\end{array}$ \\
\hline \multicolumn{6}{|c|}{ Crested wheatgrass - fairway type (Agropyron cristatum) } \\
\hline Fairway & $\begin{array}{l}\text { University of } \\
\text { Saskatchewan }\end{array}$ & L.E. Kirk & 1932 & 330 & $2,3,4$ \\
\hline Parkway & $\begin{array}{l}\text { Ag. Canada, } \\
\text { Saskatoon, SK }\end{array}$ & R.P. Knowles & 1969 & 330 & $2,3,4$ \\
\hline Kirk & $\begin{array}{l}\text { Ag. Canada, } \\
\text { Saskatoon, SK }\end{array}$ & R.P. Knowles & 1987 & 330 & $2,3,4$ \\
\hline Ruff & $\begin{array}{l}\text { ARS \& UNL, } \\
\text { Lincoln, NE }\end{array}$ & L.C. Newell & 1974 & 330 & $2,3,4,5$ \\
\hline NU-ARS-AC2 & $\begin{array}{l}\text { ARS \& UNL, } \\
\text { Lincoln, NE }\end{array}$ & K.P. Vogel & 2003 & 330 & $2,3,4,5$ \\
\hline Goliath & $\begin{array}{l}\text { Ag. Canada, } \\
\text { Saskatoon, SK }\end{array}$ & B. Coulman & 2003 & 330 & $2,3,4$ \\
\hline \multicolumn{6}{|c|}{ Crested wheatgrass - standard type (Agropyron desertorum) } \\
\hline Nordan & ARS, Mandan, ND & G. Rogler & 1953 & 330 & $2,3,4,5$ \\
\hline Summit & $\begin{array}{l}\text { Ag. Canada, } \\
\text { Saskatoon, SK }\end{array}$ & R.P. Knowles & 1953 & 330 & $2,3,4$ \\
\hline \multicolumn{6}{|c|}{ Intermediate wheatgrass (Thinopyrum intermedium; previously Agropyron intermedium) } \\
\hline Ree & $\begin{array}{l}\text { South Dakota } \\
\text { State University }\end{array}$ & C.J. Franske & 1945 & 330,250 & $2,3,4,5$ \\
\hline Nebraska 50 & $\begin{array}{l}\text { ARS \& UNL, } \\
\text { Lincoln, NE }\end{array}$ & L.C. Newell & 1950 & 330,250 & $2,3,4,5$ \\
\hline Chief & $\begin{array}{l}\text { Ag. Canada, } \\
\text { Saskatoon, SK }\end{array}$ & R.P. Knowles & 1961 & 330,250 & $2,3,4,5$ \\
\hline Oahe & $\begin{array}{l}\text { South Dakota } \\
\text { State University }\end{array}$ & J.G. Ross & 1961 & 330,250 & $2,3,4,5$ \\
\hline Greenleaf & $\begin{array}{l}\text { Ag. Canada, } \\
\text { Lethbridge, AB }\end{array}$ & R.W. Peake & 1966 & 330,250 & $2,3,4,5$ \\
\hline Slate & $\begin{array}{l}\text { ARS \& UNL, } \\
\text { Lincoln, NE }\end{array}$ & L.C. Newell & 1969 & 330,250 & $2,3,4,5$ \\
\hline Clarke & $\begin{array}{l}\text { Ag. Canada, Swift } \\
\text { Current, SK }\end{array}$ & T. Lawrence & 1980 & 330,250 & $2,3,4,5$ \\
\hline Reliant & ARS, Mandan, ND & J. Berdahl & 1991 & 330,250 & $2,3,4,5$ \\
\hline Manska & ARS, Mandan, ND & J. Berdahl & 1992 & 330,250 & $2,3,4,5$ \\
\hline Beefmaker & $\begin{array}{l}\text { ARS \& UNL, } \\
\text { Lincoln, NE }\end{array}$ & K.P. Vogel & 2003 & 330,250 & $2,3,4,5$ \\
\hline Haymaker & $\begin{array}{l}\text { ARS \& UNL, } \\
\text { Lincoln, NE }\end{array}$ & K.P. Vogel & 2003 & 330,250 & $2,3,4,5$ \\
\hline Manifest & ARS Mandan, ND & J. Berdahl & 2007 & 330,250 & $2,3,4,5$ \\
\hline \multicolumn{6}{|c|}{ Tall wheatgrass (Thinopyrum ponticum; previously Agropyron elongatum) } \\
\hline Nebraska 98526 & $\begin{array}{l}\text { ARS \& UNL, } \\
\text { Lincoln, NE }\end{array}$ & L.C. Newell & 1950 & 330,250 & $3,4,5$ \\
\hline
\end{tabular}


Table 2 (continued)

\begin{tabular}{|c|c|c|c|c|c|}
\hline \multirow{2}{*}{$\begin{array}{l}\text { Species/ } \\
\text { Cultivar }^{*}\end{array}$} & \multirow{2}{*}{$\begin{array}{l}\text { Agency or } \\
\text { University }^{*}\end{array}$} & \multirow{2}{*}{$\begin{array}{l}\text { Breeder or } \\
\text { Developer }\end{array}$} & \multirow{2}{*}{$\begin{array}{l}\text { Released } \\
\text { Year }\end{array}$} & \multicolumn{2}{|c|}{ Adaptation Region } \\
\hline & & & & Ecoregion ${ }^{\dagger}$ & $\begin{array}{l}\text { Hardiness } \\
\text { Zone }^{\ddagger}\end{array}$ \\
\hline Orbit & $\begin{array}{l}\text { Ag. Canada, Swift } \\
\text { Current, SK }\end{array}$ & T. Lawrence & 1966 & 330,250 & $3,4,5$ \\
\hline Platte & $\begin{array}{l}\text { ARS \& UNL, } \\
\text { Lincoln, NE }\end{array}$ & L.C. Newell & 1972 & 330,250 & $3,4,5$ \\
\hline \multicolumn{6}{|c|}{ Western wheatgrass (Pascopyrum smithii; previously Agropyron smithii) } \\
\hline Barton & Manhattan PMC & $\begin{array}{l}\text { R.D Lippert \& } \\
\text { H. Hackerott }\end{array}$ & 1970 & 330 & $3,4,5$ \\
\hline Rosana & Bridger PMC & $\begin{array}{l}\text { J.L. Williams \& } \\
\text { A.M. Thornburg }\end{array}$ & 1972 & 330 & $3,4,5$ \\
\hline Flintlock & $\begin{array}{l}\text { ARS \& UNL, } \\
\text { Lincoln, NE }\end{array}$ & L.C. Newell & 1975 & 330 & $3,4,5$ \\
\hline Walsh & $\begin{array}{l}\text { Ag. Canada, } \\
\text { Lethbridge, AB }\end{array}$ & $\begin{array}{l}\text { S. Smoliak } \\
\text { A. Johnston }\end{array}$ & 1982 & 330 & $2,3,4$ \\
\hline Rodan & ARS, Mandan, ND & R. Barker \& G.A. Rogler & 1983 & 330 & $2,3,4$ \\
\hline \multicolumn{6}{|c|}{ Canada Wildrye (Elymus canadensis) } \\
\hline Mandan & ARS, Mandan, ND & G.A. Rogler & 1946 & 250 & $2,3,4$ \\
\hline Homestead & $\begin{array}{l}\text { ARS \& UNL, } \\
\text { Lincoln, NE }\end{array}$ & K.P. Vogel & 2008 & 250 & 4,5 \\
\hline \multicolumn{6}{|c|}{ Russian Wildrye (Psathyrostachys juncea; previously Elymus juncea) } \\
\hline Vinall & ARS Mandan, ND & $\begin{array}{l}\text { G.A. Rogler \& } \\
\text { H.M. Schaaf }\end{array}$ & 1960 & 330 & $2,3,4$ \\
\hline Sawki & $\begin{array}{l}\text { Ag. Canada, Swift } \\
\text { Current, SK }\end{array}$ & T. Lawrence & 1963 & 330 & $2,3,4$ \\
\hline Mayak & $\begin{array}{l}\text { Ag. Canada, Swift } \\
\text { Current, SK }\end{array}$ & T. Lawrence & 1971 & 330 & $2,3,4$ \\
\hline Cabree & $\begin{array}{l}\text { Ag. Canada, } \\
\text { Lethbridge, AB }\end{array}$ & S. Smoliak & 1976 & 330 & $2,3,4$ \\
\hline Swift & $\begin{array}{l}\text { Ag. Canada, Swift } \\
\text { Current, SK }\end{array}$ & T. Lawrence & 1978 & 330 & $2,3,4$ \\
\hline Tetracan & $\begin{array}{l}\text { Ag. Canada, Swift } \\
\text { Current, SK }\end{array}$ & T. Lawrence & 1988 & 330 & $2,3,4$ \\
\hline Mankota & ARS Mandan, ND & J. Berdahl & 1991 & 330 & $2,3,4$ \\
\hline \multicolumn{6}{|c|}{ Altai wildrye (Leymus angustus; previously Elymus angustus) } \\
\hline Prairieland & $\begin{array}{l}\text { Ag. Canada, Swift } \\
\text { Current, SK }\end{array}$ & T. Lawrence & 1976 & 330 & $2,3,4$ \\
\hline Eejay & $\begin{array}{l}\text { Ag. Canada, Swift } \\
\text { Current, SK }\end{array}$ & T. Lawrence & 1989 & 330 & $2,3,4$ \\
\hline Pearl & $\begin{array}{l}\text { Ag. Canada, Swift } \\
\text { Current, SK }\end{array}$ & T. Lawrence & 1989 & 330 & $2,3,4$ \\
\hline \multicolumn{6}{|c|}{ Smooth bromegrass (Bromus inermis) } \\
\hline Superior & $\begin{array}{l}\text { Ag. Canada, } \\
\text { Saskatoon, SK }\end{array}$ & $\begin{array}{l}\text { L.E. Kirk \& } \\
\text { T.M. Stevenson }\end{array}$ & & 250 & $2,3,4$ \\
\hline Parkland & Ag. Canada, & L.E. Kirk \& & & 250 & $2,3,4$ \\
\hline
\end{tabular}


Table 2 (continued)

\begin{tabular}{|c|c|c|c|c|c|}
\hline \multirow{2}{*}{$\begin{array}{l}\text { Species/ } \\
\text { Cultivar }^{*}\end{array}$} & \multirow{2}{*}{$\begin{array}{l}\text { Agency or } \\
\text { University }^{*}\end{array}$} & \multirow{2}{*}{$\begin{array}{l}\text { Breeder or } \\
\text { Developer* }\end{array}$} & \multirow{2}{*}{$\begin{array}{l}\text { Released } \\
\text { Year }\end{array}$} & \multicolumn{2}{|c|}{ Adaptation Region } \\
\hline & & & & Ecoregion ${ }^{\dagger}$ & $\begin{array}{l}\text { Hardiness } \\
\text { Zone }^{\ddagger}\end{array}$ \\
\hline & Saskatoon, SK & T.M. Stevenson & & & \\
\hline Lincoln & $\begin{array}{l}\text { ARS \& UNL, } \\
\text { Lincoln, NE }\end{array}$ & L.C. Newell & 1942 & 250 & $3,4,5$ \\
\hline Homesteader & $\begin{array}{l}\text { South Dakota } \\
\text { State University }\end{array}$ & J.G. Ross & 1951 & 250 & $2,3,4$ \\
\hline Lyon & $\begin{array}{l}\text { ARS \& UNL, } \\
\text { Lincoln, NE }\end{array}$ & L.C. Newell & 1950 & 250 & $3,4,5$ \\
\hline Lancaster & $\begin{array}{l}\text { ARS \& UNL, } \\
\text { Lincoln, NE }\end{array}$ & L.C. Newell & 1950 & 250 & $3,4,5$ \\
\hline Carlton & $\begin{array}{l}\text { Ag. Canada, } \\
\text { Saskatoon, SK }\end{array}$ & R.P. Knowles & 1961 & 250 & $2,3,4$ \\
\hline Magna & $\begin{array}{l}\text { Ag. Canada, } \\
\text { Saskatoon, SK }\end{array}$ & R.P. Knowles & 1968 & 250 & $2,3,4$ \\
\hline Rebound & $\begin{array}{l}\text { South Dakota } \\
\text { State University }\end{array}$ & J.G. Ross & 1978 & 250 & $3,4,5$ \\
\hline Signal & $\begin{array}{l}\text { Ag. Canada, } \\
\text { Saskatoon, SK }\end{array}$ & R.P. Knowles & 1983 & 250 & $2,3,4$ \\
\hline Radisson & $\begin{array}{l}\text { Ag. Canada, Saint } \\
\text { Foy, OC \& } \\
\text { Saskatoon, SK }\end{array}$ & $\begin{array}{l}\text { J. Surprenant \& } \\
\text { R.P. Knowles }\end{array}$ & 1990 & 250 & $2,3,4$ \\
\hline Newell & $\begin{array}{l}\text { ARS \& UNL, } \\
\text { Lincoln, NE }\end{array}$ & K.P. Vogel & 2014 & 250 & $3,4,5$ \\
\hline \multicolumn{6}{|c|}{ Meadow bromegrass (Bromus riparius; previously Bromus biebersteinii) } \\
\hline Paddock & $\begin{array}{l}\text { Ag. Canada, } \\
\text { Saskatoon, SK }\end{array}$ & R.P. Knowles & 1987 & 250 & $2,3,4,5$ \\
\hline Fleet & $\begin{array}{l}\text { Ag. Canada, } \\
\text { Saskatoon, SK }\end{array}$ & R.P. Knowles & 1987 & 250 & $2,3,4,5$ \\
\hline \multicolumn{6}{|c|}{ Hybrid bromegrass (Bromus riparious $\times$ Bromus inermis) } \\
\hline AC Knowles & $\begin{array}{l}\text { Ag. Canada, } \\
\text { Saskatoon, SK }\end{array}$ & B. Coulman & 2000 & 250 & $2,3,4$ \\
\hline AC Success & $\begin{array}{l}\text { Ag. Canada, } \\
\text { Saskatoon, SK }\end{array}$ & B. Coulman & 2003 & 250 & $2,3,4$ \\
\hline \multicolumn{6}{|c|}{ Creeping foxtail (Alopecurus arundinaceus) } \\
\hline Garrison & Bismarck PMC & J. McDermand & 1963 & 330,250 & $2,3,4,5$ \\
\hline \multicolumn{6}{|c|}{$\begin{array}{l}\text { Note: The previous scientific name of some species is also listed because of changes that have been made in names to reflect new knowledge of the } \\
\text { relationships of species. } \\
\text { Ag. Canada indicates Agricultural and Agri-Food Canada followed by laboratory location; ARS, Agricultural Research Service, USDA; UNL, University o } \\
\text { Nebraska-Lincoln; PMC, NRCD-USDA Plant Materials Center preceded by PMC location. } \\
{ }^{*} \text { Other information sources: Release notices in Agronomy Journal, Crop Science, Journal of Plant Registrations; Canadian release sources; anc } \\
\text { extension papers. } \\
{ }^{+} \text {Bailey Ecoregions: } 250 \text { Prairie = tall grass prairie, } 330 \text { Temperate Steppe = short \& mid-grass prairie. } \\
{ }^{*} \text { The Plant Hardiness Zones are those of the } 2012 \text { USDA Hardiness Zone Map at: http://planthardiness.ars.usda.gov/PHZMWeb/. }\end{array}$} \\
\hline
\end{tabular}

seed lots, which were sent to Saskatoon at a later date from Mandan, North Dakota. The first crested wheatgrass cultivar developed for use in North America was "Fairway", which was released in 1932. It was bred by L.E. Kirk (Fig.
2), who at the time of release was a Professor at the University of Saskatchewan. ${ }^{15,18}$

There was a similar development pattern for smooth bromegrass, which was first introduced into the United States 
Table 3. Cultivars of native grasses developed and released for use in the Northern Plains of the United States and Canada, their principal developing agency and location, plant breeder or developer, and adaptation region $n^{34,37,38,39}$

\begin{tabular}{l|l|l|l|l|l|}
\hline $\begin{array}{l}\text { Species/ } \\
\text { Cultivar }\end{array}$ & $\begin{array}{l}\text { Agency \& } \\
\text { University }^{*}\end{array}$ & $\begin{array}{l}\text { Breeder or } \\
\text { Developer }^{*}\end{array}$ & Release & \multicolumn{2}{|l}{ Adaptation Region } \\
\end{tabular}

Big bluestem (Andropogon gerardii; previously Andropogon furcatus)

\begin{tabular}{|c|c|c|c|c|c|}
\hline Kaw & $\begin{array}{l}\text { Kansas State University } \\
\& \text { Manhattan PMC }\end{array}$ & K.L. Anderson & 1950 & 250, Мз30 & Lower 5, 6 \\
\hline Champ & $\begin{array}{l}\text { ARS \& UNL, } \\
\text { Lincoln, NE }\end{array}$ & L.C. Newell & 1963 & 250, Мз30 & 4, upper 5 \\
\hline Pawnee & $\begin{array}{l}\text { ARS \& UNL, } \\
\text { Lincoln, NE }\end{array}$ & L.C. Newell & 1963 & 250, M330 & 5, upper 6 \\
\hline Bonilla & Bismarck PMC & $\begin{array}{l}\text { J. McDermand, E. Jacobson, } \\
\text { R.J. Haas, R.E. Barker }\end{array}$ & 1987 & 250, М330 & 3,4 \\
\hline Bison & $\begin{array}{l}\text { ARS Mandan, ND \& } \\
\text { Bismarck PMC }\end{array}$ & $\begin{array}{l}\text { R.E. Barker, R.J. Haas, } \\
\text { J. Berdahl, E. Jacobson }\end{array}$ & 1989 & 250, М330 & $2,3,4$ \\
\hline Sunnyview & $\begin{array}{l}\text { South Dakota } \\
\text { State University }\end{array}$ & A. Boe & 1998 & 250, М330 & $\begin{array}{l}\text { Lower 2, 3, } \\
4\end{array}$ \\
\hline Bonanza & $\begin{array}{l}\text { ARS \& UNL, } \\
\text { Lincoln, NE }\end{array}$ & K.P. Vogel & 2004 & 250, M330 & 5, upper 6 \\
\hline Goldmine & $\begin{array}{l}\text { ARS \& UNL, } \\
\text { Lincoln, NE }\end{array}$ & K.P. Vogel & 2004 & 250, Мз30 & 5,6 \\
\hline
\end{tabular}

Sand bluestem (Andropogon hallii; previously Andropogon gerardii var. incanescens)

\begin{tabular}{|c|c|c|c|c|c|}
\hline Garden & $\begin{array}{l}\text { Scottsbluff \& } \\
\text { Manhattan PMC }\end{array}$ & NRCS PMC staff & 1960 & 330 & Lower 4,5 \\
\hline Goldstrike & $\begin{array}{l}\text { ARS \& UNL, } \\
\text { Lincoln, NE }\end{array}$ & L.C. Newell & 1973 & 330 & Lower 4, 5 \\
\hline \multicolumn{6}{|c|}{ Indiangrass (Sorghastrum nutans) } \\
\hline $\begin{array}{l}\text { Nebraska } \\
54\end{array}$ & Farmer & H. Hummel & 1957 & 250, М330 & 5 , upper 6 \\
\hline Holt & $\begin{array}{l}\text { ARS \& UNL, } \\
\text { Lincoln, NE }\end{array}$ & L.C. Newell & 1960 & 250, М330 & 4, upper 5 \\
\hline Oto & $\begin{array}{l}\text { ARS \& UNL, } \\
\text { Lincoln, NE }\end{array}$ & L.C. Newell & 1970 & 250, M330 & Lower 5, 6 \\
\hline Tomahawk & Bismarck PMC & $\begin{array}{l}\text { J. McDermand, E. Jacobson, } \\
\text { R.J. Haas, R.E. Barker }\end{array}$ & 1988 & 250, М330 & 3,4 \\
\hline Chief & $\begin{array}{l}\text { ARS \& UNL, } \\
\text { Lincoln, NE }\end{array}$ & K.P. Vogel & 2008 & 250, M330 & 4, upper 5 \\
\hline Scout & $\begin{array}{l}\text { ARS \& UNL, } \\
\text { Lincoln, NE }\end{array}$ & K.P. Vogel & 2008 & 250, М330 & 5 \\
\hline Warrior & $\begin{array}{l}\text { ARS \& UNL, } \\
\text { Lincoln, NE }\end{array}$ & K.P. Vogel & 2008 & 250, М330 & Lower 5,6 \\
\hline \multicolumn{6}{|c|}{ Switchgrass (Panicum virgatum) } \\
\hline Blackwell & Manhattan PMC & PMC staff & 1944 & 250, М330 & Lower 5, 6 \\
\hline $\begin{array}{l}\text { Nebraska } \\
28\end{array}$ & $\begin{array}{l}\text { ARS \& UNL, } \\
\text { Lincoln, NE }\end{array}$ & L.C. Newell & 1949 & 250, М330 & $\begin{array}{l}3,4 \text {, upper } \\
5\end{array}$ \\
\hline Pathfinder & ARS \& UNL, & L.C. Newell & 1967 & 250, Мз30 & 4,5 \\
\hline
\end{tabular}


Table 3 (continued)

\begin{tabular}{|c|c|c|c|c|c|}
\hline \multirow{2}{*}{$\begin{array}{l}\text { Species/ } \\
\text { Cultivar }^{*}\end{array}$} & \multirow{2}{*}{$\begin{array}{l}\text { Agency \& } \\
\text { University }^{*}\end{array}$} & \multirow{2}{*}{$\begin{array}{l}\text { Breeder or } \\
\text { Developer* }\end{array}$} & \multirow{2}{*}{$\begin{array}{l}\text { Release } \\
\text { Year }\end{array}$} & \multicolumn{2}{|c|}{ Adaptation Region } \\
\hline & & & & Ecoregion $^{\dagger}$ & $\begin{array}{l}\text { Hardiness } \\
\text { Zone }^{\ddagger}\end{array}$ \\
\hline & Lincoln, NE & & & & \\
\hline Summer & $\begin{array}{l}\text { South Dakota } \\
\text { State University }\end{array}$ & J.G. Ross & 1963 & 250, M330 & 4,5 \\
\hline Sunburst & $\begin{array}{l}\text { South Dakota } \\
\text { State University }\end{array}$ & A. Boe \& J.G. Ross & 1983 & 250, M330 & $\begin{array}{l}\text { Lower 2, 3, } \\
4\end{array}$ \\
\hline Trailblazer & $\begin{array}{l}\text { ARS \& UNL, } \\
\text { Lincoln, NE }\end{array}$ & K.P. Vogel & 1984 & 250, M330 & 4,5 \\
\hline Forestburg & $\begin{array}{l}\text { Bismarck PMC \& } \\
\text { ARS, Mandan ND }\end{array}$ & $\begin{array}{l}\text { J. McDermand, E. Jacobson, } \\
\text { R. J. Haas, R.E. Barker }\end{array}$ & 1987 & 250, М330 & 3,4 \\
\hline Dacotah & $\begin{array}{l}\text { ARS-Mandan, ND } \\
\text { \& Bismarck PMC }\end{array}$ & $\begin{array}{l}\text { R.E. Barker, G. Rogler, } \\
\text { R.J. Haas, E.T. Jacobson }\end{array}$ & 1989 & 250, М330 & $\begin{array}{l}2,3 \text {, upper } \\
4\end{array}$ \\
\hline Shawnee & $\begin{array}{l}\text { ARS \& UNL, } \\
\text { Lincoln, NE }\end{array}$ & K.P. Vogel & 1995 & 250, M330 & $4,5,6$ \\
\hline Liberty & $\begin{array}{l}\text { ARS \& UNL, } \\
\text { Lincoln, NE }\end{array}$ & K.P. Vogel & 2013 & 250, М330 & 5,6 \\
\hline \multicolumn{6}{|c|}{ Little bluestem (Schizachyrium scoparium; previously Andropogon scoparius) } \\
\hline Blaze & $\begin{array}{l}\text { ARS \& UNL, } \\
\text { Lincoln, NE }\end{array}$ & L.C. Newell & 1967 & 250,330 & $\begin{array}{l}\text { Lower } 4 \text { \& } \\
5\end{array}$ \\
\hline Camper & $\begin{array}{l}\text { ARS \& UNL, } \\
\text { Lincoln, NE }\end{array}$ & L.C. Newell & 1973 & 250,330 & $\begin{array}{l}\text { Lower } 4 \& \\
5\end{array}$ \\
\hline \multicolumn{6}{|c|}{ Sideoats grama (Bouteloua curtipendula) } \\
\hline Trailway & $\begin{array}{l}\text { ARS \& UNL, } \\
\text { Lincoln, NE }\end{array}$ & $\begin{array}{l}\text { L.C. Newell \& } \\
\text { E.C. Conard }\end{array}$ & 1956 & 250,330 & $\begin{array}{l}\text { Lower } 4 \text { \& } \\
5\end{array}$ \\
\hline Butte & $\begin{array}{l}\text { ARS \& UNL, } \\
\text { Lincoln, NE }\end{array}$ & $\begin{array}{l}\text { L.C. Newell \& } \\
\text { E.C. Conard }\end{array}$ & 1956 & 250,330 & $\begin{array}{l}\text { Lower } 4 \& \\
5\end{array}$ \\
\hline Pierre & Bismarck PMC & J. McDermand & 1965 & 250,330 & 3,4 \\
\hline \multicolumn{6}{|c|}{ Blue grama (Bouteloua gracilis) } \\
\hline Bad River & $\begin{array}{l}\text { Bismarck PMC } \\
\text { (an ecotype } \\
\text { release) }\end{array}$ & PMC Staff & 1996 & 330 & 3,4 \\
\hline \multicolumn{6}{|c|}{ Sand lovegrass (Eragrostis tricodes) } \\
\hline $\begin{array}{l}\text { Nebraska } \\
27\end{array}$ & $\begin{array}{l}\text { ARS \& UNL, } \\
\text { Lincoln, NE }\end{array}$ & $\begin{array}{l}\text { L.C. Newell \& } \\
\text { E.C. Conard }\end{array}$ & 1949 & 250,330 & $\begin{array}{l}\text { Lower } 4 \& \\
5\end{array}$ \\
\hline \multicolumn{6}{|c|}{ Prairie Sandreed (Calamolvilfa longifolia) } \\
\hline Goshen & Bridger PMC & $\begin{array}{l}\text { A.A. Thornburg, J.R. Stroh, } \\
\text { J.G. Scheetz }\end{array}$ & 1976 & 330 & $\begin{array}{l}\text { Lower } 4 \text { \& } \\
5\end{array}$ \\
\hline Pronghorn & $\begin{array}{l}\text { ARS \& UNL, } \\
\text { Lincoln, NE }\end{array}$ & K.P. Vogel \& L.C. Newell & & 330 & $\begin{array}{l}\text { Lower } 4 \& \\
5\end{array}$ \\
\hline
\end{tabular}

Note: The previous scientific name of some species is also listed due to changes that have been made in names to reflect new knowledge of the relationships of species. Ag. Canada indicates Agricultural and Agri-Food Canada followed by laboratory location; ARS, Agricultural Research Service, USDA; UNL, University of Nebraska-Lincoln; PMC, NRCS-USDA Plant Materials Center preceded by PMC location.

* Other information sources: Release notices in Agronomy Journal, Crop Science, Journal of Plant Registrations; Canadian release sources; and extension papers.

${ }^{\dagger}$ Bailey (2009) Ecoregions: 250 Prairie = tall grass prairie, 330 Temperate Steppe = short \& mid-grass prairie (M330 = midgrass prairie which is not defined by Bailey).

* The Plant Hardiness Zones are those of the 2012 USDA Hardiness Zone Map at: http://planthardiness.ars.usda.gov/PHZMWeb/. 


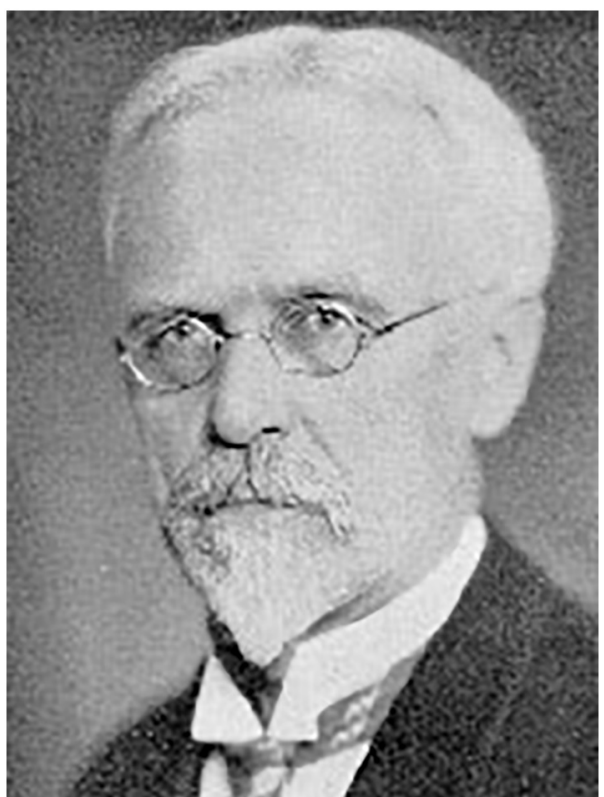

Figure 1. Professor Niels Hansen, South Dakota State University. Dr Hansen conducted plant exploration and plant introduction work for the US Department of Agriculture in the late 1800s and early 1900s, including the collection and introduction of the crested wheatgrasses and smooth bromegrasses.

and Canada in the 1880s. ${ }^{19}$ The first introduction was by the California Experiment Station in 1884, which distributed seed to other experiment stations. Major introductions were from central Europe, including Hungary, northern Germany, and the Russian collections of N.E. Hansen. ${ }^{7,16}$ By the late 1890s, bromegrass was being grown in the Midwest and Great Plains of the United States and Canada. Bromegrass identified as "Hungarian" bromegrass was sown on the University of Nebraska Experiment Station farm at Lincoln, Nebraska in 1897 in a small field. ${ }^{20}$ Seed and forage yields

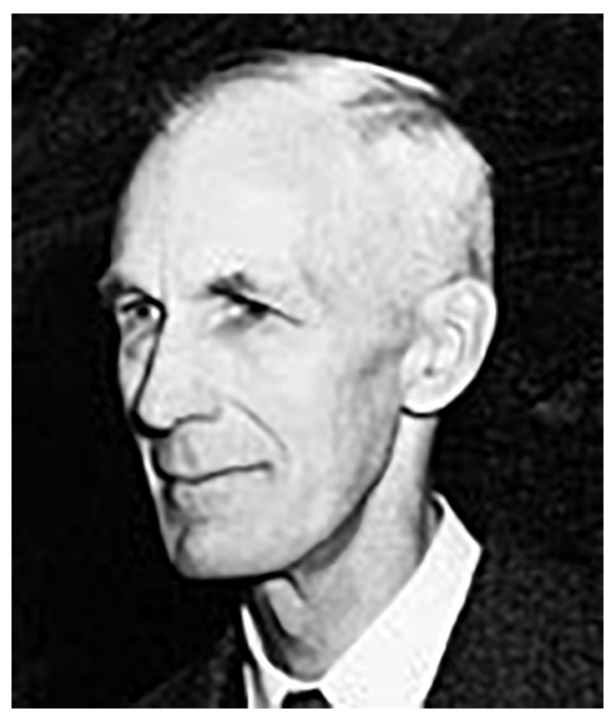

Figure 2. Lawrence Kirk, Canadian scientist and grass breeder, who developed the first crested wheatgrass cultivar grown in North America. were determined. In 1898, a 16-acre pasture was established that was used in grazing trials by cattle and horses. In his summary, Lyon noted that its advantage over the native prairie grasses was that it became green fully a month earlier in the spring and remained green later into the autumn. ${ }^{20} \mathrm{He}$ indicated that it was safe and widely adaptable. Similar results were being obtained from other experiment stations. ${ }^{7}$ The initial spread and distribution of smooth bromegrass in North America were based on the simple increase and distribution of the introduced strains from experiment stations to farmers. During the 30-year period from the late 1890s to the early 1930s, agronomic information was obtained on the management of smooth bromegrass at locations throughout the prairie regions of the United States and Canada. The old stands and pastures that survived the droughts of the 1930s were used for both seed production in the initial revegetation efforts and the germplasm basis for the initial breeding programs.

Because of this pioneering work, the basic pattern or system was developed that was used in subsequent grass breeding and agronomic research. The development steps included collecting a large number of germplasms or accessions of species, evaluating the accessions in uniform trials, selecting the best accessions for advanced evaluation or breeding work, developing the agronomic information needed to use the species in grazing lands, and releasing tested materials as cultivars for certified seed production by seed growers. The germplasm of a species is the total genetic variability of the species that breeders use to develop cultivars. The germplasm accessions used by breeders are seed or plants collected from specific plants, sites, or regions.

The crested wheatgrass and bromegrass seed initially used after the great drought to reseed rangeland, pastures, and abandoned cropland was from seed increase fields or pastures that had been planted to the best plant introduction of these species. Other than Fairway crested wheatgrass in Canada, there were no cultivars of these species. The initial plantings were from different plant introductions, and in the case of crested wheatgrass, two different species with the same common name. Because there were no purity standards or seed certification systems, the seedlots available to farmers and ranchers essentially became a mixed-up hodgepodge of the species for which no adaptation and production information was available. Grass breeding work was initiated to improve grass species for use in agricultural production systems by developing cultivars with known adaptation and production capabilities, which could be produced in a systematic manner via seed certification.

\section{Plant Breeding and Plant Materials Programs Agricultural Research Service-USDA Grass Breeding Programs in the Northern Plains \\ Northern Great Plains Laboratory, Mandan, North Dakota}

The Northern Great Plains Research Laboratory, USDA, was established in 1912. ${ }^{21}$ As described previously, grass evaluation work had begun by 1915 but this work largely consisted of species comparison and agronomic research. The 
first grass breeding was initiated in 1936 when George Rogler was transferred by the USDA to the station to begin grass breeding work. The first cultivar he developed was Nordan crested wheatgrass, which was released in 1953. It became the most widely used crested wheatgrass in the United States for decades. It was a major improvement over the common seedlots of crested wheatgrass that had been previously available in the United States. He developed or $\mathrm{co}^{-}$ developed cultivars of western wheatgrass, Russian wildrye, Canada wildrye, and switchgrass (Tables 2 and 3). From the mid-1950s until his retirement in 1973, Rogler devoted most of his research effort to developing improved range and forage management practices to improve livestock production per acre, which are regarded as among his most major accomplishments. ${ }^{21}$ During this period, geneticist Herbert Schaaf conducted most of the grass breeding efforts until he was transferred to another location in 1969. He codeveloped the cultivar Vinall Russian wildye with Rogler. ${ }^{21}$

The grass breeding work at Mandan was terminated in 1969 when Herbert Schaaf was transferred, but was reinstated and funded by Congress in $1973 .{ }^{21}$ Dr Reed Barker reported to Mandan in 1972 and re-initiated the grass breeding work (R. Barker, personal communication, March 2018). The grass breeding program at Mandan was expanded in 1976 when another geneticist, Dr John Berdahl, was hired. ${ }^{21}$ Reed Barker's breeding work focused primarily on crested and western wheatgrasses with some work on native grasses in cooperation with the Bismarck, North Dakota Plant Materials Center. John Berdahl's work focused primarily on intermediate wheatgrasses and Russian wildrye. Dr Barker transferred to Corvallis, Oregon in 1988. Geneticist Ian Ray was hired in 1990 to refill the position vacated by Reed Barker, but he transferred away from the Northern Great Plains Laboratory in 1993. Dr Berdahl retired in 2006 and the long-term grass breeding program at Northern Great Plains Laboratory was terminated at that time. ${ }^{21}$ The grass cultivars developed by Barker and Berdahl included Rodan western wheatgrass, Mankota Russian Wildrye, and Reliant, Manska, and Manifest intermediate wheatgrass (Table 2). These grass cultivars have been widely used in the Dakotas. They also participated with the Bismarck Plant Materials Center staff in the development and release of Bison and Bonilla big bluestem, Tomahawk indiangrass, and two switchgrass cultivars (Table 3).

USDA-ARS and University of Nebraska-Lincoln, Lincoln, Nebras$k a$

The grass breeding program at Lincoln, Nebraska was established as a cooperative USDA-ARS and University of Nebraska project in $1936{ }^{12,22}$ USDA provided the scientist along with operating funds and research equipment while the University of Nebraska-Lincoln (UNL) provided technical and secretarial support, some operating funds, and experiment station land and greenhouse and laboratory facilities. Although university technical support and operating funds are no longer provided, university facilities and land are still made available to ARS scientists at Lincoln in 2018.

The first USDA grass breeder at Lincoln was Laurence C. Newell (Fig. 3) who started the grass breeding program in 1936. He was a native Nebraskan who had received an A.B. degree from Hastings College and was a high school teacher and principal when he developed an interest in native grasses (L. C. Newell, Dec. 1985, personal communication). He left his teaching career and went to the University of Nebraska as a graduate assistant where he obtained an MS in agronomy in 1933 with a research emphasis on native grasses. From 1933 to 1936, he held several positions including assisting with some of the original USDA-SCS native grass collections in the Great Plains. He completed his PhD degree at UNL in

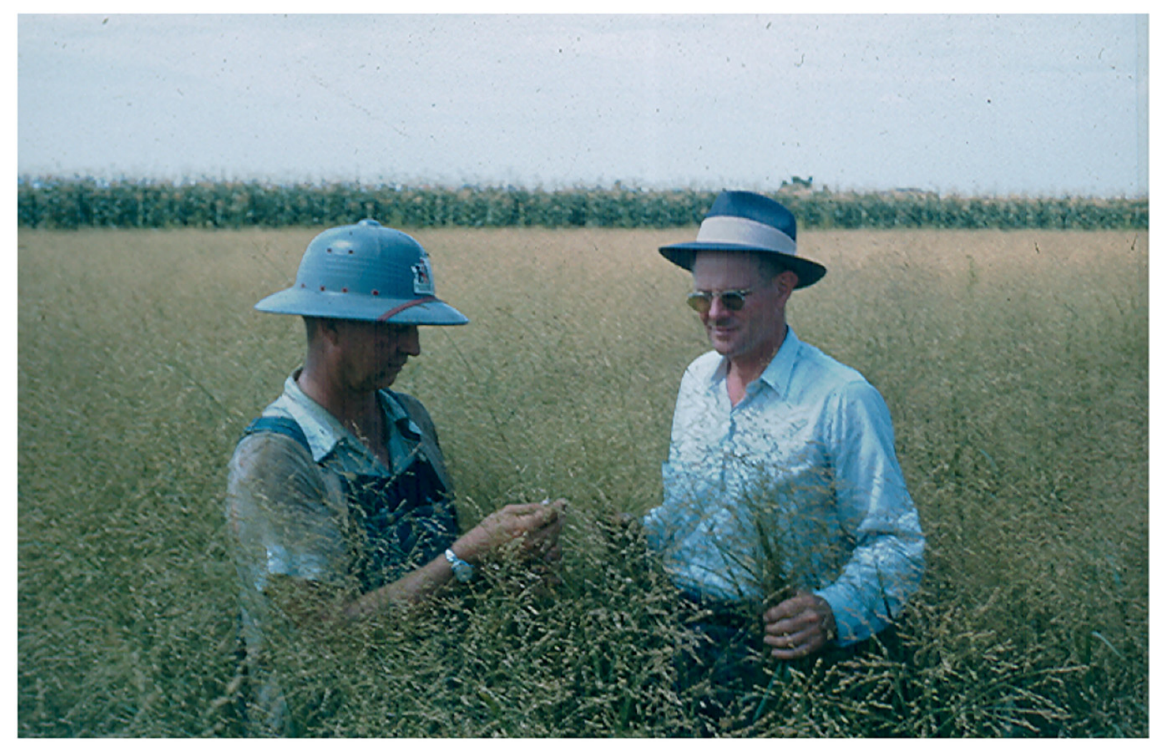

Figure 3. USDA grass breeder Dr Laurence C. Newell (right) and seed producer Harold Hummel in one of the first switchgrass seed production fields. In addition to his breeding work on native grasses, L.C. Newell also worked on bromegrass and wheatgrasses, and his bromegrass cultivar Lincoln was the most widely grown bromegrass in the United States. 
1940 while working as a USDA scientist. L.C. Newell was a very productive grass breeder. He developed cultivars of bromegrass, crested, western, tall, and intermediate wheatgrass, switchgrass, big bluestem, indiangrass, little bluestem, side-oats grama, and sandlovegrass (Tables 2 and 3). He developed the bromegrass cultivar "Lincoln," which was released to seed growers in 1940 and became the most widely used bromegrass cultivar in the United States. ${ }^{19}$ These cultivars were used in the adjacent Midwest states as well as Great Plains states. In addition to his cultivar development accomplishments, Dr Newell also did research on the daily and seasonal distribution of pollen by grasses and their dissemination distance on which grass seed certification isolation distance are still based for many species. Dr Newell retired in the spring of 1975 after 39 years of work as a grass breeder in Lincoln.

Ken Vogel (senior author) was hired to replace Dr Newell in September of 1974 shortly after he had completed his $\mathrm{PhD}$ in plant breeding at Nebraska. He used many of the breeding populations that had been developed by L.C. Newell, as well as prairie collections and new plant introductions to develop the second or third generation of grass cultivars for many of the same species (Tables 2 and 3). Most of the cultivars that he developed were bred for improved forage digestibility in addition to having improved forage yield and other traits. This was possible due to cooperative arrangements with the Animal Science Department at UNL, which gave ARS scientists at Lincoln access to rumen fistulated animals so that they could use rumen fluid in laboratory digestion trials. In cooperation with UNL animal scientists, Lincoln ARS scientists also were able to conduct replicated pasture trials to quantify the improvements in animal gains per acre made by developing cultivars with improved digestibility and forage yields. Trailblazer switchgrass, Bonanza and Goldmine big bluestem, Scout and Warrior indiangrass, Manska intermediate wheatgrass, and Newell smooth bromegrass were all released based on data from grazing trials. Grazing trials can add 5 years to the development period of a new cultivar (Table 1), but greatly improve acceptance by producers. Beginning in 1990, Vogel began working on developing switchgrass into a biomass energy grass for use in the Northern Plains and the Midwest, and Lincoln became a lead location in the United States for this work. The first biomass energy type switchgrass cultivar, Liberty, was released in 2013. Ken Vogel retired at the end of 2013. Dr Serge Edme was transferred to replace him in 2015 and is continuing the grass breeding work at Lincoln.

\section{Land Grant Universities}

South Dakota State University, Brookings, South Dakota

Staff at South Dakota State University (SDSU, formerly South Dakota State College of Agriculture and Mechanic Arts) were involved early in grass improvement work through the germplasm collection work of N.E. Hansen as described previously. The South Dakota Experiment Station was also involved in the initial crested wheatgrass forage and persistence evaluation trials as well as the first grazing trials. ${ }^{4}$ The first grass improvement work per se was conducted by agronomist Clifford J. Franzke, who was on the staff at SDSU from 1924 to 1964 (A. Boe, personal communication, June 2018). As a result of his grass evaluation work, he identified the intermediate wheatgrass accession PI (Plant Introduction) 98568 as a superior strain and had it released without any further selection as the cultivar Ree. It was the first intermediate wheatgrass cultivar released in North America. He also worked on many other crops and developed a forage sorghum that had reduced hydrocyanic acid ( $\mathrm{HCN})$ potential (A. Boe, personal communication, June 2018).

The first person hired specifically to conduct grass breeding research at SDSU was James G. Ross, who worked at SDSU from 1947 to 1981 (A. Boe, personal communication, June 2018). He was born and raised in Alberta, Canada, and received his $\mathrm{PhD}$ in plant genetics from the University of Wisconsin. He developed cultivars of smooth bromegrass, intermediate wheatgrass, and switchgrass (see Tables 2 and 3). Ross conducted some of the first breeding work on improving forage digestibility in cool-season grasses adapted to the Northern Plains. He also did extensive germplasm collections of switchgrass, big bluestem, and indiangrass that served as the germplasm basis for breeding work on those native species. In addition to his research work, Dr Ross also taught at SDSU since he had both a teaching and research assignment.

Dr Arvid Boe was hired as the SDSU grass breeder to replace J.G. Ross after completing his $\mathrm{PhD}$ work under Ross at SDSU (A. Boe, personal communication, June 2018). He has been conducting grass breeding and genetics research at SDSU from 1981 to the present. He has released Sunburst switchgrass and Sunnyview big bluestem cultivars. He has conducted breeding and genetics research on developing switchgrass into a biomass energy crop since 1999 and has worked on prairie cordgrass (Spartina pectinata) for bioenergy. His work on regional adaptation of grass cultivars with other grass breeders has greatly improved the adaptation information on grasses for the Northern Plains and the Northern Midwest states. Dr Boe has had both teaching and research assignments at SDSU.

\section{Other Land Grant Universities}

The other Land Grant University in the Northern Great Plains in which there has been a continuous long-term grass breeding program is the cooperative grass USDA and University of Nebraska project at Lincoln, which has been described previously. There have been grass genetics projects at other land grant universities in the Northern Plains, but their support and duration has not been consistent and as a result their development of grass cultivars for use on grazing lands has not been as effective as the programs at SDSU and Nebraska. It simply takes long-term, stable funding to effectively develop good cultivars of perennial grasses for use on grazing lands. It should be noted, however, that A.E. Aldous of Kansas State University began working on big bluestem in 1928 and smooth bromegrass in $1930 .{ }^{12}$ The work on big bluestem did lead to the release of Kaw big bluestem, which can be grown in the southern part of Nebraska but is mostly used in the Southern Great Plains. 


\section{USDA Plant Materials Centers}

A comprehensive 494-page history of the plant materials program of the Natural Resource Conservation Service (NRCS, formerly the Soil Conservation Service or SCS), USDA, has been recently been published by W. Curtis Sharp, who was the NRCS National Plant Materials Specialist from 1985 to $1993 .{ }^{11}$ This summary has been extracted from that history. The first work on plant materials for erosion control began in 1933 when the Soil Erosion Service (SES), US Department of Interior, was established. In 1933, SES nurseries were established at different sites in the United States to produce large numbers of plants and seed for use in revegetation. These nurseries were incorporated into the Soil Conservation Service when it was established. Charles R. Enlow was the first director of the SES plant nurseries and developed the initial guidelines. Franklin Crider replaced Enlow as director of the SCS Plant Materials Program in 1936.

Dr Franklin Crider (Fig. 4) was a native of South Carolina and received a BS from Clemson University, a MS from University of Minnesota, and an honorary $\mathrm{PhD}$ from the University of Arizona in $1936 .{ }^{11}$ He became a staff member of the Horticulture Department at the University of Arizona in 1918 and later became its department head. In 1924, he became the Director of the Boyce Thompson Southwest Arboretum. While at the Southwest Arboretum, he initiated a large-scale revegetation program on a degraded rangeland site in Arizona. This project involved collecting and evaluating plant materials and establishing production nurseries for use in producing seed for the revegetation effort. This work was

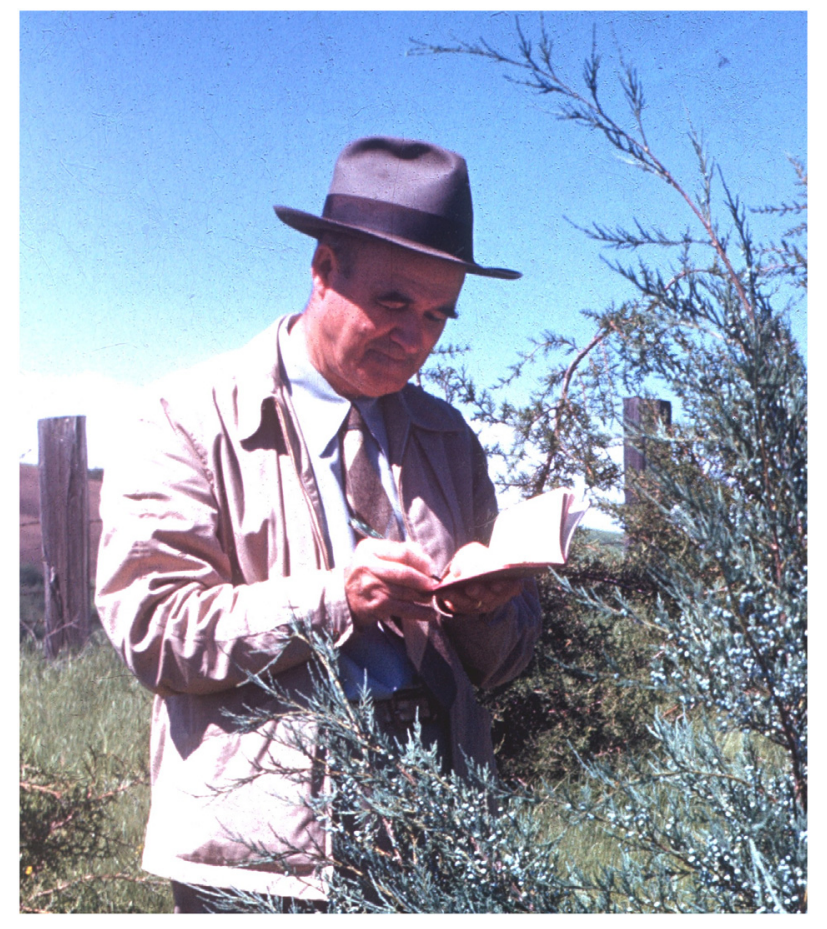

Figure 4. Dr Franklin J. Crider became director of the plant materials programs of the Soil Conservation Service (now Natural Resource Conservation Service), USDA, in 1936. He developed the basic procedures and policies of the Plant Materials Centers, many of which are still being used. the model on which subsequent federal revegetation work by the USDA-SCS was based. ${ }^{11} \mathrm{He}$ left the Southwest Arboretum to become head of the SCS plant materials programs in Washington, DC. Shortly after he assumed leadership of the plant materials program, Dr Crider revamped the initial guideless and developed the policies and basic procedures of the SCS Plant Materials Program, which were used for decades. The policies and procedures are detailed in SCS Field Memorandum \#SCN-4, which was issued in March 1936. Dr Crider headed the plant materials national program until 1939, when he transferred to Beltsville, Maryland to head up the National Observational Nursery. He retired in January 1952. He is regarded as the founder of the Plant Materials Program.

Initially two types of SCS nurseries, production and observation, were established. Production nurseries were set up to collect and produce seed and plants for use in large-scale conservation plantings. These nurseries were closed in 1954 and their function was turned over to the seed industry, which had developed by that time. The observation nurseries' function was to assemble, evaluate, and turn over the best material to the production nurseries. After 1954, the observation nurseries became the SCS Plant Materials Centers. The functions of the Plant Materials Centers (PMCs) were to identify specific needs for new or improved plant materials, collect or assemble plant materials with emphasis on native plants, evaluate the plant materials, and assist in facilitating the commercial production of the best plant materials. They also were to advise research agencies on the need for research and development. The latter function is a requirement because by statute, the PMCs are not authorized to conduct research, which must be done by the research agencies of USDA or cooperating universities. For this reason, all the PMCs evaluation work is done by "observation" and in many cases in unreplicated evaluation nurseries. However, this restriction was avoided in some work by cooperating with USDA-ARS or university scientists.

The collection and evaluation work of the PMCs is essentially Phase 1 or the first step of a perennial grass breeding program (Table 1). This initial step in a grass breeding program is known as the Ecotype or Naturalized Strain Selection Breeding System. ${ }^{23}$ In contrast to the PMCs' procedures, research-based grass breeding programs such as those of USDA-ARS and universities use data on forage yield and other traits to make selections and then intermate the best selected plants to develop improved populations for desired traits. They also conduct multiple generations of breeding to make improvements. Despite the handicap of being required to use only observational information to select superior accessions and not being able to do follow-up breeding work, staff at PMCs have been able to identify some superior plant materials that were released as cultivars. These cultivars were evaluated under field conditions following their selection and increase at the PMCs.

In 1994, a change was made in the Plant Materials Manual that enabled PMCs to release pre-variety plant material. ${ }^{11}$ The Association of Official Seed Certifying Agencies (ASOCA) recognizes three types of pre-variety plant material, which are Source Identified Pre-variety, Selected Pre-variety, and Tested 
Pre-variety. The Source Identified Pre-variety is produced by the simple increase of plant materials from known sources or sites without any type of testing or selection. The Selected Pre-Variety consists of progeny from plants that have been phenotypically (observationally) selected from known source germplasm nurseries and have been increased in a manner to ensure genetic purity and stability. These are the two main types of Pre-variety releases that have been made by the PMCs. There are no follow-up field evaluations. The Tested Pre-variety consists of the progeny of plants whose parentage has been tested and has proven genetic superiority. This type of Pre-variety release is not used by the PMCs because of lack of research authority. For all three of the Pre-variety types of plant materials, a seed certification program following ASOCA guidelines is required. The use of the Prevariety program addressed a need to get local ecotype material into seed certified programs because cheating also can occur in the native plant "local ecotype" seed business. However, as indicated by Sharp ${ }^{11}$ and Booth and Vogel, ${ }^{24}$ there are problems with the program due to some of its questionable ecological assumptions and the lack of testing.

There are three NRCS PMCs whose plant materials are used in the Northern Great Plains. It does need to be pointed out that the PMCs, in addition to perennial grasses, also did work on trees, shrubs, and forbs which were developed and used for conservation purposes.

\section{Bismarck Plant Materials Center}

This Center had its initial start as a US Department of Interior Production (USDI) nursery in 1933 and was located at Mandan, North Dakota. ${ }^{11}$ Through a somewhat complicated series of administrative steps involving the USDI, USDA, and the North Dakota Association of Conservation Districts, the observation nursery ended up on the other side of the Missouri River as the Bismarck PMC. The Bismarck Center serves the states of South Dakota, North Dakota, and Minnesota. The Bismarck PMC has been instrumental in the release or co-release of cultivars of both cool-season and warm-season grass cultivars (see Tables 1 and 2). The Bismarck PMC also has served as the source of the Foundation class of seed for grass cultivars codeveloped by the ARS scientists stationed at Mandan. The pre-variety releases are not listed except for Bad River blue grama. The Bismarck PMC managers listed in order of service were: George Rogler, Arthur Ferber, Buck Worthington, John McDermand, George Kary, Lee Hines, Sheridan Dronen, David Lorenz, Russell Haas, Dwight Tober, and Wayne Duckworth. ${ }^{11}$ John McDermand, Erling Jacobson, and Russel Haas are listed as a developers or codevelopers of several grass cultivars (Tables 1 and 2).

\section{Bridger Plant Materials Center}

This Center located at Bridger, Montana was first authorized in 1959 and serves the states of Wyoming and Montana, which means that it serves several unique ecoregions, one of which is the plains regions of these states. ${ }^{11}$ It has been instrumental in the release of grasses for use in both the intermountain area and the plains area of these states. Only the cultivars developed by the Bridger PMC of grasses typically used in the plains regions of these states are reported in Tables 1 and 2. The Bridger Plant Materials Center managers listed in order of service were: Ashley Thornburg, James Stroh, John Scheetz, Mark Majerus, Roger Hybner, and Joseph Scianna. ${ }^{11}$ Ashley Thornberg codeveloped several grass cultivars.

\section{Manhattan Plant Materials Center}

This Center was one of the first SCS production and observation nurseries that were started in $1935 .{ }^{11} \mathrm{It}$ also went through a series of administrative and management changes over the decades of its existence. At its peak when its production nursery was the most active, it had over 150 employees. The Manhattan nurseries were closed by SCS in 1953 and the observation nursery component became the Manhattan Plant Materials Center, which initially was operated under USDA contract by Kansas State University. In 1965, SCS resumed its operation of the PMC with Erling Jacobson as its manager. The Manhattan PMC is responsible for Kansas, Nebraska, northern Oklahoma, and eastern Colorado. Because of the USDA grass breeding program at Lincoln, Nebraska, most of its grass development work has been focused on Kansas and Oklahoma. It has had some notable people on its staff, including Donald Cornelius, who did significant work on seed production methods for native grasses. Two of the most widely used grass cultivars developed by the Manhattan PMC for use in the Northern Plains were Barton western wheatgrass and Blackwell switchgrass, which was the first switchgrass cultivar released. The Manhattan PMC managers listed in order of service were: William Giles, Donald Cornelius, Charles Swingel, Fred Eshbaugh, Clarence Swallow, Erling Jacobson, John Dickerson, Robert Dayton, Gary Fine, and Richard Wynia.

\section{Canadian Breeding Programs}

Grass breeding work in the Northern Plains region was initiated earlier in Canada than the United States ${ }^{12}$ and has been conducted at three locations: Saskatoon and Swift Current, Saskatchewan, and Lethbridge, Alberta. The first official grass breeding work was initiated in 1922 by L.E. Kirk, who at that time was a professor at the University of Saskatchewan. ${ }^{25}$ The grass breeding work at Saskatoon was transferred to the Canada Department of Agriculture (now Agriculture and Agri-Food Canada [AAFC]) in 1931 with the establishment of the Dominion Forage Crops Laboratory. ${ }^{25}$ It and other AAFC laboratories at Saskatoon were merged in the 1950s to become the Saskatoon Research Centre. In 1931, Dr Kirk left Saskatoon for a series of administrative positions. When he retired in 1947, he was Chief of the Plant Industry Branch of the Agricultural Division of the United Nations. ${ }^{18}$

Grass breeding work at Saskatoon was conducted by Dr Robert P. Knowles from 1941 to $1985 .{ }^{25} \mathrm{He}$ made major breeding contributions by developing improved crested and intermediate wheatgrass and smooth and meadow bromegrass cultivars (Table 1). He also made significant contributions to science in his genetics and breeding research. Dr Knowles was inducted into the Saskatchewan Hall of Fame (https://www. 
sahf.ca/inductees/k/robert_patrick_knowles.html) based on his breeding and plant genetics research. After the retirement of Dr Knowles, the grass breeding work at Saskatoon was carried out by S. Wright (1986-1993) and then by Dr Bruce Coulman from 1993 to the present. ${ }^{25}$ In 2005, Dr Coulman transferred to the University of Saskatchewan, so the grass breeding project at Saskatoon is now a cooperative program between AAFC and the university. He has developed smooth $\mathrm{x}$ meadow bromegrass hybrid cultivars that are currently being widely used in Canada as well as a new crested wheatgrass cultivar (Table 1).

Grass breeding was initiated at the Swift Current Laboratory in 1947 by D.H. Heinrichs. ${ }^{25}$ In 1954, Dr Thomas Lawrence was hired to work exclusively on grass breeding for the Swift Current region, which is the driest area of Saskatchewan. ${ }^{25}$ Dr Lawrence developed improved cultivars of Russian and Altai wildrye and intermediate and tall wheatgrass (Table 1). Based on his breeding and research accomplishments, Dr Lawrence was also inducted into the Saskatchewan Hall of Fame (https://www.sahf.ca/inductees/ 1/thomas_lawrence.html). After Dr Lawrence's retirement in 1989, a reduced breeding program was carried out by Paul Jefferson until 2006. ${ }^{25}$ Currently at Swift Current, work is being carried out on ecological varieties of native Canadian grass species by M.P. Schellenberg. ${ }^{25}$ This work is a collaborative effort with Ducks Unlimited Canada to provide plant materials for habitat restoration. ${ }^{26}$ These ecological varieties are similar to the Pre-variety plant materials being developed in the United States by the PMCs. Several grass varieties that are used in the plains region of Canada have been developed at the Lethbridge Research Center including western and intermediate wheatgrass and Russian wildrye cultivars (Table 1). Cultivars of other grass species have also been developed at the Lethbridge Laboratory but are better adapted to the intermountain regions of western Canada. Principal scientists involved in grass cultivar testing and development for the northern plains area at Lethbridge were S. Smoliak and A. Johnston.

\section{Native vs. Introduced Grasses}

Crested wheatgrass and smooth bromegrass are not regarded favorably by everyone even though they saved millions of acres of land from severe erosion and remain economically important grasses in both pasture and rangelands. ${ }^{27,28}$ Most of the criticism is based on the fact that they are not native species and that pure stands of the species lack biological diversity. Native species are considered by many ecologists to be more desirable than the introduced grasses. Smooth bromegrass and crested wheatgrass are viewed by some as invaders of native rangeland and remnant prairie sites. Personal observation and published reports ${ }^{29,30}$ indicate that neither grasses invade grasslands that are well managed and in good condition. Bromegrass will invade disturbed areas and poorly managed native grasslands, especially in the tallgrass prairie ecoregion. Crested wheatgrass is very noninvasive and usually does not spread from where it is planted. The soil quality of long-term crested wheatgrass fields or range sites has been reported to be below that in adjacent, unplowed prairie. ${ }^{27,28}$ The fact that the sites seeded to crested wheatgrass had been plowed and eroded before they were seeded to crested wheatgrass is often ignored or remains a confounding factor in these reports. Other reports indicate that grazed crested wheatgrass and native prairies produce similar amounts of root biomass. ${ }^{31}$ The crested wheatgrass and smooth bromegrass are now naturalized species of North America. Their use in revegetation and conservation saved the soil on millions of acres of land. They will continue to be used in production agriculture and in restoring degraded rangelands that have been invaded by noxious weeds.

\section{Accomplishments and the Future}

As indicated previously, the work of a small number of plant breeders and plant materials specialists in the United States and Canada has resulted in an array of superior grass cultivars for use in the Northern Great Plains (Tables 2 and 3). Not all of these cultivars are still in seed production as some of the older cultivars have been replaced by newer, superior cultivars. Statistics on the acres seeded to these cultivars in the United States are simply not available because this data is not tracked by the USDA's National Agricultural Statistics Service (NASS) in the United States. In addition, some of the land planted to grasses in the Northern Great Plains has been plowed and converted to crops and then replanted to grasses depending on crop prices and farm programs. Some information is available on the amount of certified seed produced by seed growers for some cultivars, and by estimating seeding rates it is possible to estimate the acres seeded, but a full accounting using this method exceeds the scope of this report. Since virtually all the grasses seeded in the Northern Great Plains since the late 1930s originated from the programs described previously in this report, the acres planted to these grasses include almost all seeded grasslands in the Northern Plains except for small prairie restoration areas that have been planted to local ecotypes. In Canada, the grass cultivars developed by Canadian breeders were reported to have helped enable the cattle population in western Canada to triple. ${ }^{18}$

The most productive perennial grass breeding programs were the ones with stable long-term funding and with breeders who were able to conduct work over several decades, which is essential for a perennial plant breeding program. There are still opportunities for significant improvements in each of the grass species to make them more economically valuable in production systems. As examples, switchgrass and big bluestem cultivars with improved forage digestibility and forage yields developed by Vogel at Nebraska increased beef cattle gains per year by up to 60 pounds per acre in multiple year grazing trials without any additional costs. ${ }^{32,33}$ The economic value of these gains will vary with cattle prices.

The factors that lead to the economic and environmental conditions that lead to the severe land degradation problems following the 1930s drought still exist in the Northern Plains. 
High grain prices result in grasslands being plowed if the owners think that they can make more money on crops than cattle and grass. Grain crop breeders are continuing to make improvements in grain crop yields, which need to be matched by improvements in grazing land profitability to keep land in perennial grasses. This can be done by both developing improved grasses and legumes for use in grazing lands and improvements in grazing land management. Several of the long-term grass breeding programs in the Northern Plains have been terminated, downgraded, or converted to other research thrusts. In addition, grant funding in the United States has replaced the stable, long-term funding in many areas of agricultural research. Instead of government funding problems dealing with overproduction of grain crops that typically follows major grassland plow-ups, modest investments in long-term research programs in grass breeding and grazing land management would appear to be a good investment.

\section{Acknowledgments}

Authors thanks Dr Bruce Coulman for the information provided on Canadian grass breeders, Dr Arvid Boe for information on the grass breeding work at South Dakota State University, Dr Reed Barker for information on the USDAARS grass breeding work at Mandan, ND, and W. Curtis Sharp (retired USDA-NRCS) for his extensive history of the USDA Plant Materials program (see references) and previous personal communications and photos.

\section{References}

1. BAiley, R.G. 2009. Ecosystem geography: from ecoregion to sites. 2nd ed. New York, NY, USA: Springer. 251 p.

2. EDWARDS, E.E. 1948. The settlements of grasslands. Grass. Yearbook of agriculture 1948. Washington, DC, USA: US Government Printing Office. p. 16-25.

3. Morison, S.E. 1965. The Oxford history of the American people. New York, NY, USA: Oxford Press. 1150 p.

4. Dillman, A.C. 1946. The beginnings of crested wheatgrass in North America. Journal of the American Society of Agronomy 38:237-250.

5. Campbell, J.B. 1971. The Swift Current Research Station 1920-70. Historical series No. 6. Ottawa, Canada: Canada Department of Agriculture. Available at: http://publications.gc. ca/collections/collection_2016/aac-aafc/agrhist/A54-2-6-1971eng.pdf, Accessed date: 30 August 2018.

6. LORENZ, R.J. 1983. Introduction and early use of crested wheatgrass in the Northern Great Plains. In: \& Johnson KL, editor. Crested wheatgrass: its values, problems, and myths. Symposium Proceedings Utah State University; Logan, UT, USA; 3-7 Oct 1983. Logan, UT, USA: Utah State University. p. 9-20.

7. Vogel, K.P. 2004. Humans, climate, and plants: the migration of crested wheatgrass and smooth bromegrass to the Great Plains of North America. In: Werner D, editor. Biological resources and migration. Berlin: Springer-Verlag. p. 35-45.

8. Egan, T. 2006. The worst hard time. New York, NY, USA: First Mariner Books.

9. Wallace, H.A. 1936. Report of the Secretary of Agriculture to the President of the United States, Washington, 10 December 1935. Yearbook of agriculture 1936. Washington, DC, USA: US Government Printing Office. p. 1-117.
10. HuRT, L.C. 1948. The Northern Great Plains. For a better range management. Grass. Yearbook of agriculture 1948. Washington, DC, USA: US Government Printing Office. p. 486-491.

11. SHARP, W.C. 2013. Conservation plants. A USDA success story. Middletown, DE, USA: William Sharp. 494 p.

12. Vinall, H.N., AND M.A. HeIn. 1937. Breeding miscellaneous grasses. Yearbook of agriculture 1937. Washington, DC, USA: US Government Printing Office. p. 1032-1102.

13. Cornelius, D.R. 1950. Seed production of native grasses under cultivation in eastern Kansas. Ecological Monographs 20:1-29.

14. Masters, R.A., R.B. Mitchell, K.P. Vogel, and S.S. WALLER. 1993. Influence of improvement practices on big bluestem and indiangrass seed production in tallgrass prairies. Journal of Range Management 46:183-187.

15. Rogler, G.A., And R.J. Lorenz. 1983. Crested wheatgrassEarly history in the United States. Journal of Range Management 36:91-93.

16. TAYLOR, H.J. 1941. To plant the prairies and the plains. The life and work of Niels Ebbesen Hansen, XII. BIOS. p. 1-72.

17. Griesbach, R.J. 2013. 150 Years of research at the United States Department of Agriculture. Plant introduction and breeding. Washington, DC, USA: US Department of Agriculture, Agricultural Research Service. 23 p.

18. Dale-Burnett, L.L. 2006. Saskatchewan agriculture: lives past and present. In: Miazgar B, editor. Regina Saskatchewan. Canada: Canadian Plains Research Center, University of Regina.

19. Vogel, K.P., K.J. Moore, and L.W. Moser. 1996. Bromegrasses. In: Moser LE, Buxton D, \& Casler MD, editors. Coolseason forage grasses. Agronomy monograph, Madison, WI, USA: American Society of Agronomy, Crop Science Society of America, Soil Science Society of America. p. 535-567.

20. LyON, T.L. 1899. Hungarian brome grass. Nebraska Agricultural Experiment Station Bulletin 61(XII):35-63.

21. FRANK, A.B. 2013. The taming of the prairie: a century of agricultural research at the Northern Great Plains Research Laboratory, Mandan, ND. Washington, DC, USA: US Department of Agriculture, Agricultural Research Service. Available at: https://www.ars.usda. gov/is/np/TamingofthePrairie/TamingofthePrairie.pdf, Accessed date: 30 August 2018.

22. Frolick, E.F., and R.J. Graham. 1987. The University of Nebraska-Lincoln College of Agriculture. The first century. Lincoln, NE, USA: The Board of Regents, University of Nebraska. Available at: http://digitalcommons.unl.edu/ianrhistory, Accessed date: 30 August 2018.

23. Vogel, K.P., And J.F. Pedersen. 1993. Breeding systems for crosspollinated perennial grasses. Plant Breeding Reviews 11:251-274.

24. Booth, D.T., And K.P. Vogel. 2006. Revegetation priorities. Rangelands 28:24-30.

25. BRUCE, C., AND P. JeFFerson. 2013. Ninety years of perennial forage grass breeding for the Canadian prairie provinces. In: Michalk DL, Millar GD, Badgery WB, \& Broadfoot KM, editors. Revitalizing grasslands to sustain our communities Proceedings of the 22nd International Grassland Congress, Sydney, Australia 15-19 Sept. 2013. p. 290. Available at: http://internationalgrasslands.org/files/ igc/publications/2013/proceedings-22nd-igc.pdf, Accessed date: 30 August 2018.

26. Coulman, B.E., J.G. McLeod, P.G. Jefferson, and B. Wark. 2008. Development of pre-variety germplasm of Canadian native grass species. Multifunctional grasslands in a changing worldProceedings of the 21st International Grassland Congress and 8th International Rangeland Congress, Hohhot, China 29 June to July 5, 2008. p. 447. Available at: http://internationalgrasslands.org/ files/igc/publications/2008/multifunctional-grasslands-vol-2.pdf, Accessed date: 30 August 2018. 
27. LesicA, P., And T.H. DeLuca. 1996. Long-term harmful effects of crested wheatgrass on Great Plains grassland ecosystems. Journal of Soil and Water Conservation 51:408-409.

28. Christian, J.M., And S.D. Wilson. 1999. Long-term ecosystem impacts of an introduced grass in the northern Great Plains. Ecology 80:2397-2407.

29. Blankenspoor, G.W., and J.K. May. 1996. Alien smooth brome (Bromus inermis Leyss) in a tallgrass prairie remnant: seed bank, seedling establishment, and growth dynamics. Natural Areas Journal 16:289-294.

30. Broersma, K., M. Krzic, D.J. Thompson, and A.A. Romke. 2000. Soil and vegetation of ungrazed crested wheatgrass and native rangelands. Canadian Journal of Soil Science 80:411-417.

31. Krzic, M., K. Broersma, D.J. Thomson, and A.A. Bomke. 2000. Soil properties and species diversity of grazed crested wheatgrass and native rangelands. Journal of Range Management 53:353-358.

32. Casler, M.D., And K.P. Vogel. 1999. Accomplishments and impact from breeding for increased forage nutritional value. Crop Science 39:12-20.

33. Mitchell, R.B., K.P. Vogel, T.J. Klopfenstein, B.E. Anderson, And R.A. Masters. 2005. Grazing evaluation of big bluestems bred for improved forage yield and digestibility. Crop Science 45:2288-2292.

34. Alderson, J., And W.C. Sharp. 1994. Grass varieties in the United States. US Department of Agriculture, Soil Conservation Service (USDA-ARS) Agricultural Handbook 170. Washington, DC, USA: US Government Printing Office.

35. Asay, K.H., And K.B. Jensen. 1996. Wheatgrasses. In: Moser LE, Buxton D, \& Casler MD, editors. Cool-season forage grasses. Agronomy monographMadison, WI, USA: American Society of Agronomy, Crop Science Society of America, Soil Science Society of America. p. 691-724.
36. Asay, K.H., And K.B. Jensen. 1996. Wildryes. In: Moser LE, Buxton D, \& Casler MD, editors. Cool-season forage grasses. Agronomy monographMadison, WI, USA: American Society of Agronomy, Crop Science Society of America, Soil Science Society of America. p. 725-748.

37. Boe, A., K.H. Keeler, G.A. Normann, and S.L. Hatch. 2004. The indigenous bluestems of the western hemisphere and gambagrass. In: Moser LE, Burson BL, \& Sollenberger LE, editors. Warm-season (C4) grasses. Agronomy monographMadison, WI, USA: American Society of Agronomy, Crop Science Society of America, Soil Science Society of America. p. 873-908.

38. Mitchell, R., AND K.P. Vogel. 2004. Indiangrass. In: Moser LE, Burson BL, \& Sollenberger LE, editors. Warm-season (C4) grasses. Agronomy monographMadison, WI, USA: American Society of Agronomy, Crop Science Society of America, Soil Science Society of America. p. 937-953.

39. Vogel, K.P. 2004. Switchgrass. In: Moser LE, Burson BL, \& Sollenberger LE, editors. Warm-season (C4) grasses. Agronomy monographMadison, WI, USA: American Society of Agronomy, Crop Science Society of America, Soil Science Society of America. p. 561-588.

Authors are Emeritus Professor of Agronomy and Horticulture, Univ. of Nebraska-Lincoln and retired Research Geneticist USDA-ARS, Lincoln, NE. USA 68583-0937 (kvogel1@unl. edu, Vogel); and Research Rangeland Management Specialist, USDA-ARS, Great Plains Northern Research Laboratory, Mandan, ND, USA 58554 (john.hendrickson@ars.usda.gov, Hendrickson). 